\title{
Cytokeratin 18-associated Histone 3 Modulation in Hepatocellular Carcinoma: A Mini Review
}

\author{
YEN-CHANG CLARK LAI ${ }^{1}$, CHIUNG-CHI CHENG ${ }^{2,3}$, YIH-SHYONG LAI ${ }^{2}$ and YI-HSIANG LIU ${ }^{2,4}$ \\ ${ }^{1}$ Department of Pathology, Kaohsiung Medical University Hospital, Kaohsiung, Taiwan, R.O.C.; \\ ${ }^{2}$ Department of Pathology, Chang Bing Show Chwan Memorial Hospital, Changhua, Taiwan, R.O.C.; \\ ${ }^{3}$ Center for General Education, Providence University, Taichung, Taiwan, R.O.C.; \\ ${ }^{4}$ Department of Pathology, Tzu Chi University, Hualien, Taiwan, R.O.C.
}

\begin{abstract}
Unstable cytokeratins are associated with tumor transformation in the development of human hepatocellular carcinoma. We previously demonstrated that the cytokeratin 18 was modulated and that a histone H3-specific modification occured, among members of the histone family, during the development of human hepatocellular carcinoma. Evidence suggested that the modification of histone $\mathrm{H} 3$ was highly correlated with the modulation of cytokeratin 18 and probably plays an important role in tumorigenesis of hepatocytes. Aberrant expression of histone deacetylase leading to imbalance between acetylation and deacetylation of histones may exhibit regulatory roles in tumor transformation. Recently we found that overexpression of histone deacetylase-1 and hypoacetylation of histone $\mathrm{H3}$ were associated with hepatocellular carcinoma. The underlying roles of histone $\mathrm{H} 3$ modulation are discussed in this mini-review article.
\end{abstract}

Hepatocellular carcinoma (HCC) is a cancer with high incidence and mortality in Taiwan. HCC cells exhibit different morphological features in divergent shapes and having pleomorphic nuclei from normal liver cells. Microtubules (MTs), intermediate filaments (IFs), and microfilaments (MFs) are major components of the

This article is freely accessible online.

Correspondence to: Dr. Yi-Hsiang Liu, Department of Pathology, Chang Bing Show Chwan Memorial Hospital, 6, Lugong Road, Lugang Town, Changhua County 505, Taiwan, R.O.C. Tel: +886 47813888 (Ext. 71181 71182), Fax: +886 47073235, e-mail: ysliu53@gmail.com

Key Words: Cytokeratin, hepatocellular carcinoma, histone H3, histone deacetylase 1, review. cytoskeleton that are essential to maintain the integrity of eukaryotic cells (1). Proper cross-linking structures among these cytoskeletal proteins are crucial for intracellular architectures and normal cellular morphology. Plectin exhibits the binding sites accessible to IFs, MTs and MFs rendering the interaction with a variety of cytoskeletal components to maintain the integrity of the cytoskeletal network (2). The rope-like IFs have a mean diameter of $10 \mathrm{~nm}$ and mainly play a role in maintaining the shape and mechanical integrity of a cell (3). In human hepatocytes, a cytokeratin (CK) pair composed of CK8 (type II, $52 \mathrm{kD}$ ) and CK18 (type I, $45 \mathrm{kD}$ ) renders IFs (4). CKs are required for maintaining the integrity of hepatocytes (5). Altered expression of keratin genes were associated with chronic hepatitis, increased hepatocyte fragility and decreased bile secretion (6).

In eukaryotic nucleus, the organization and packaging of DNA are achieved by addition of histone proteins to form chromatin. Post-translational modifications of histones, such as acetylation, phosphorylation, methylation and ADPribosylation, frequently alter the structure of chromatin (7). Epigenetic modifications of histone, especially acetylation status, altering the chromatin structure are involved in gene expression and further intervened in the pathogenesis of cancer. Two types of enzymes, including histone acetyltransferases (HATs) and histone deacetylases (HDACs), affect the acetylation status of histones. Recently it was reported that alterations in the activities of HDACs, as well as aberrant acetylation of histone, lead to diseases including cancer (8). HDACs catalyze histone deacetylation and repress transcription of specific genes, such as p21, resulting in cell-cycle activation and cell proliferation (9).

The investigation of histones in our laboratory originated in a study of CK18, in which we found that CK18 was coimmunoprecipitated with histone $\mathrm{H} 3$ in human $\mathrm{HCC}$, but not in normal liver. We speculated that both histone $\mathrm{H} 3$ and CK18 were modulated in HCC, which was shown in the co- 
immunoprecipitation of our experiments. Our opinion could be important for exploring the tumor transformation in the development of human HCC. However, by reviewing the literature to date, we found that the relation between histone modification and cytoskeleton modulation was not investigated. In this review, we display our series of studies proposing towards CK18-associated histone 3 modulations in HCC.

\section{Disorganization of CK18 Triggered by Plectin Deficiency in Human HCC}

We investigated the CK molecules of liver cells and asserted the modulation of $\mathrm{CK} 18$ during tumor transformation in HCC tissue and hepatoma cell lines in our previous studies $(10,11)$. We also found that deficiency of plectin (a crosslinking protein in cytoskeleton) in liver cells triggered the modulation of CK18. The disorganization of CK18 and altered levels of CK18 expression is associated with plectin knock-down $(12,13)$ and degradation $(14,15)$ in liver cells. In consequence, liver cells showed cytoskeletal augmentation and pleomorphic changes. Furthermore, we discovered plectin-deficient human hepatic cells exhibited higher cell motility in association with increased focal adhesion kinase activity that was comparable to the properties of invasive HCC (16). Recently, we reported that higher cell motility and collective cell migration are associated with deficiency in plectin and increased expression of E-cadherin in hepatoma cells (17). Plectin may be involved in the regulation of cell motility that is related to the invasiveness of HCC. We also suggest that hepatoma cells with plectin deficiency and high level of E-cadherin expression are more sensitive to sorafenib treatment (17).

\section{Histone H3 Co-immunoprecipitated with CK18 in Human HCC}

In addition to the modulation of CK18, a group of proteins with a molecular weight range between 12.4-18.4 kDa (mainly $14 \mathrm{kDa}$ ) were also found in $\mathrm{HCC}$. These low molecular-weight proteins were designated as HCC CK in our previous reports due to their specific association with CK18 in immunoprecipitation experiments $(10,11)$. It was, at first, thought that HCC CK was derived from CK18 in HCC. However, the following evidence demonstrating different origins of HCC CK and CK18 were found in our studies. Firstly, use of an anti-CK18 antibody failed to detect HCC CK on western blot assays; in contrast, HCC CK was detected by the use of an anti-histone antibody, indicating discrepancy in antigenicity. Secondly, N-terminal sequence mapping of HCC CK revealed high homology to that of histone rather than CK18. However, not all histones were found in the HCC CK fraction isolated from human HCC.
Western blotting assay only detects histone $\mathrm{H} 3$ in $\mathrm{HCC}$ while the signals of $\mathrm{H} 1, \mathrm{H} 2 \mathrm{~A}, \mathrm{H} 2 \mathrm{~B}$, and $\mathrm{H} 4$ were not present (18). Therefore, we proposed a hypothesis that the structure of chromosomes and the organization of nuclei were unstable and more fragile in $\mathrm{HCC}$, leading to histone proteins accessible to extraction procedures, and in consequence the histone $\mathrm{H} 3$ was co-immunoprecipitated with CK18. The hypothesis was verified by series of experiments using anti-CK18 and antihistone $\mathrm{H} 3$ antibody for immunoprecipitation in human liver and HCC tissues, respectively. The data suggested that histone $\mathrm{H} 3$ and CK18 was co-immunoprecipitated and simultaneously exist in HCC cells, while this phenomenon was not observed in normal liver cells (19). It is widely accepted that expression of CK18 affects nuclear organization then further impacts the integrity and stability of HCC cell nuclei. Reorganization of cytoskeleton perturbing the function of nuclear matrix was the suggested mechanism since Barboro et al. reported that dramatic changes in the expression of both the nuclear matrix and IF proteins occur during transformation of rat hepatocyte nodules (20). Our latest study also showed that nuclear pleomorphism of hepatoma cells is correlated with the cytoplasmic disorganization of cytoskeleton caused by plectin deficiency; irregular nuclear shape resembled pleomorphic nuclei in cancer cells were observed in the plectin-knockdown liver cells (21).

\section{Hypoacetylation of Histone $\mathrm{H3}$ in Human HCC}

The roles of histone modification, especially acetylation and deacetylation, in the development of cancer have been recently investigated. HDACs exhibit important roles in histone modification and chromatin remodeling. Deacetylation of histones activates gene expression involved in the molecular pathway of HCC tumorigenesis was reported by Buurman et al. (22). Abnormal expression of HDACs causes aberrant level of histone acetylation and in consequence may lead to tumor transformation in human HCC. Our evidence showed that aberrant acetylation was associated with the modulation of histone $\mathrm{H} 3$ in human HCC. We found in human HCC tissues, the level of histone $\mathrm{H} 3$ and HDAC1 was increased whereas the content of acetylated histone $\mathrm{H} 3$ ( $\mathrm{AH} 3$ ) was reduced in comparison to those in normal liver tissues (23). Therefore, we suggested that hypoacetylated modification of histone $\mathrm{H} 3$ is derived from enhanced expression of HDAC1 in human HCC. In a separate report by Farooq et al., altered expression of HDAC1 in HCC was found to regulate the critical factors related to the processes of liver carcinogenesis (24). In our studies, data supported that hypoacetylation of histone $\mathrm{H} 3$ accompanied with enhanced expression of HDAC1 was involved in the development of human HCC (23). The mechanism of HDACs involved in tumor transformation of $\mathrm{HCC}$ is still unclear. Aberrant regulation of cell cycle and apoptosis were the 


\section{Liver Cell HCC Cell}

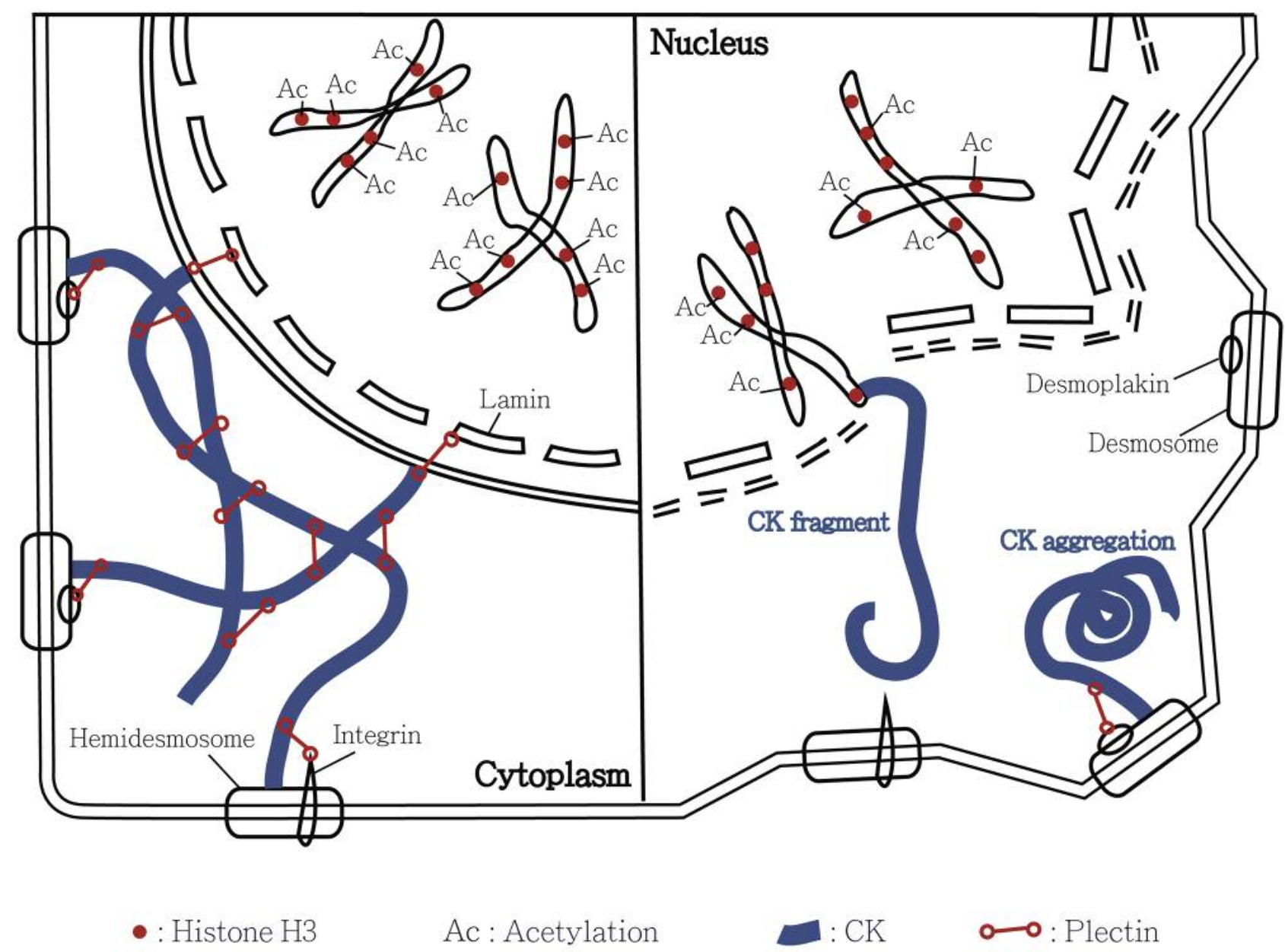

Figure 1. Illustrative model for the involvement of cytokeratin-associated histone 3 modulation in the tumor transformation of hepatocellular carcinoma (HCC). In normal liver cells, cytokeratin is maintained by the cytokeratin-connected plectin. The filamentous meshwork attaches to the lamin, integrin and desmoplakin forming stable cell architecture and nuclear membrane. Normally acetylation of histone H3 is under equilibrium. Size and shape of liver cells are consistent. However, deficiency in plectin and cytokeratin meshwork results in collapse of HCC cells. In consequence, the cytokeratin filaments may aggregate or become short fragments. Overexpression of histone deacetylase-1 results in hypoacetylation of histone H3 in HCC. It leads to unstable cell membrane, nuclear envelope and chromosome and ultimately to cell transformation. We hypothesize that some cytokeratin fragments connect to histone H3 in HCC cells. This model explains co-immunoprecipitation of histone H3 and CK18 in human HCC.

proposed mechanisms. Buurman et al. found that in liver cells, microRNA-449 specifically binds to $c$-MET mRNA, can promote apoptosis and inactivates cell proliferation. It has been found that enhanced expression of HDAC1-3 was associated with reduced level of microRNA-449. Consequently, proliferation-inhibiting effect mediated by microRNA-449 was suppressed (22). Sun et al. also reported that microRNA-34a regulates HDAC1 expression to affect the proliferation and apoptosis of hepatocellular carcinoma. Low microRNA-34a expression and high HDAC1 expression in $\mathrm{HCC}$ are closely related to the occurrence and development of HCC (25). The correlation of the HDAC expression with tumor progression was reported but the completion was still controversial. Rikimaru et al. advocated that in human HCC, increased expression of HDAC1 was associated with higher invasiveness, lower differentiation, and poor prognosis (26). In contrast, Zhao et al. claimed that expression of HDAC1 was significantly higher in HCC tissues than that in adjacent tissues, but there was no statistical difference between HDAC1 with tumor stage (27). 


\section{Applications of HDAC Inhibitor for the Therapy of HCC}

Recently, HDAC inhibitors are being applied under the rationale of cancer therapy. Many investigations applying HDAC inhibitors for treating HCC are undergoing and many articles have been published worldwide. A novel hydroxamic acid-derived HDAC inhibitor, LBH589, suppresses proliferation and metastasis of HCC by mediating inhibitory effect on gankyrin/stat3/akt pathway (28). The pan-deacetylase inhibitor, panobinostat, affects angiogenesis in HCC models by regulating connective tissue growth factor expression (29). Vaproic acid exhibits HDAC4-inhibitory activity arresting $\mathrm{HCC}$ cell growth via increasing acetylation of histone $\mathrm{H} 4$ and suppressing notch signaling (30). Droxinostat, a HDAC inhibitor, was found to suppress HDAC3 expression and induce acetylation of histones $\mathrm{H} 3$ and $\mathrm{H} 4$. This compound can inhibit cell proliferation and induce apoptosis in HCC cell lines by activating mitochondrial apoptotic pathways (31). A novel histone deacetylase inhibitor, MPT0G009 was reported to be a potential new candidate drug for HCC therapy. The research team found that MPT0G009 can induce cell apoptosis and synergistic anticancer activity with tumor necrosis factorrelated apoptosis-inducing ligand against HCC (32). Considering the immunotherapy, suberoylanilide hydroxamic acid (SAHA, a HDAC inhibitor) regulates the expression of microRNA-17-92 cluster then epigenetically elevates the level of major histocompatibility complex class I-related chain molecules A (MICA) and maintains complex component 7 (MCM7) in hepatoma. The outcome is to enhance the sensitivity of HCC to natural killer cell-mediated lysis (33). Combined pharmaceutical treatment has also become a popular issue in cancer therapy. Sorafenib inhibits SAHAinduced NF-kB activity that regulates the expression of oncogenic proteins. Combined with sorafenib, SAHA exhibits enhanced therapeutic efficacy against HCC in vitro and in vivo through the mechanism suppressing ERK/NF-KB signaling pathway (34). A phase-I dose-escalation study of the mTOR inhibitor sirolimus and the HDAC inhibitor vorinostat in patients with advanced malignancy was reported. The authors reported that this therapy is a safe and efficacious for several cancer types including HCC (35). This evidence supports that HDAC inhibitors reveal emerging applications for treating advanced HCC.

\section{Conclusion}

In human HCC, our investigational data suggested discrepancy in immunospecificity of CK18 between HCC and normal liver tissues. We further specified the human $\mathrm{HCC}$-associated changes in histone $\mathrm{H} 3$. A great amount of evidence indicates that expression of histone $\mathrm{H} 3$ was highly related to the modulation of CK18 and likely plays an important role in tumorigenesis of human HCC. Furthermore, we confirmed that CK18 modulation and nuclear pleomorphism in HCC was correlated with the plectin deficiency. Our studies verified the previous observation that overexpression of HDAC1 in HCC catalyzes histone deacetylation and results in hypo-acetylated histone $\mathrm{H} 3$. Therefore, activation of HDAC1 is likely involved in tumor transformation of human HCC. In addition to surgical resection and trans-arterial chemoembolization, application of HDAC inhibitors provides an option for the treatment of human HCC. Figure 1 summarizes our concept regarding the CK18-associated histone 3 modulation involved in the tumor transformation of human HCC.

\section{Acknowledgements}

The Authors greatly appreciate Miss Chen, You-Yin and Miss Cheng, Jia-Min for their skillful laboratory assistance.

\section{References}

1 Huber F, Boire A, Lopez MP and Koenderink GH: Cytoskeletal crosstalk: when three different personalities team up. Curr Opin Cell Biol 32: 39-47, 2015.

2 Castanon MJ, Walko G, Winter L and Wiche G: Plectinintermediate filament partnership in skin, skeletal muscle, and peripheral nerve. Histochem Cell Biol 140: 33-53, 2013.

3 Goldman RD, Cleland MM, Murthy SN, Mahammad S and Kuczmarski ER: Inroads into the structure and function of intermediate filament networks. J Struct Biol 177: 14-23, 2012.

4 Omary MB, Ku NO and Toivola DM: Keratins: guardians of the liver. Hepatology 35: 251-257, 2002.

5 Loranger A, Duclos S, Grenier A, Price J, Wilson-Heiner M, Baribault $\mathrm{H}$ and Marceau N: Simple epithelium keratins are required for maintenance of hepatocyte integrity. Am J Pathol 151: 1673-1683, 1997.

6 Omary MB and $\mathrm{Ku} \mathrm{NO}$ : Intermediate filament proteins of the liver: emerging disease association and functions. Hepatology 25: 1043-1048, 1997.

7 Strahl BD and Allis CD: The language of covalent histone modifications. Nature 403: 41-45, 2000.

8 Timmermann S, Lehrmann H, Polesskaya A and Harel-Bellan A: Histone acetylation and disease. Cell Mol Life Sci 58: 728-736, 2001.

9 Archer SY and Hodin RA: Histone acetylation and cancer. Curr Opin Genet Dev 9: 171-174, 1999.

$10 \mathrm{Su} \mathrm{B}$, Pei RJ, Yeh KT, Hsu YH and Lai YS: Could the cytokeratin molecule be modulated during tumor transformation in hepatocellular carcinoma? Pathobiology 62: 155-159, 1994.

11 Liu YH, Pei RJ, Yeh CC, Lee KY, Yeh KT, Hsu YH, Ho CC and Lai YS: The alteration of cytokeratin 18 molecule and its mRNA expression during tumor transformation in hepatoma. Res Commun Mol Pathol Pharmacol 96: 243-253, 1997.

12 Cheng CC, Liu YH, Ho CC, Chao WT, Pei RJ, Hsu YH, Yeh KT, Ho LC, Tsai MC and Lai YS: The influence of plectin deficiency on stability of cytokeratin 18 in hepatocellular carcinoma. J Mol Histol 39: 209-216, 2008. 
13 Liu YH, Cheng CC, Ho CC, Chao WT, Pei RJ, Hsu YH, Ho LC, Shiu BH and Lai YS: Plectin deficiency on cytoskeletal disorganization and transformation of human liver cells in vitro. Med Mol Morphol 44: 21-26, 2011.

14 Liu YH, Cheng CC, Ho CC, Chao WT, Pei RJ, Hsu YH, Yeh KT, Ho LC, Tsai MC and Lai YS: Degradation of plectin with modulation of cytokeratin 18 in human liver cells during staurosporine-induced apoptosis. In Vivo 22: 543-548, 2008.

15 Liu YH, Ho CC, Cheng CC, Chao WT, Pei RJ, Hsu YH and Lai YS: Cytokeratin 18-mediated disorganization of intermediate filaments is induced by degradation of plectin in human liver cells. Biochem Biophys Res Commun 407: 575-580, 2011.

16 Cheng CC, Lai YC, Lai YS, Hsu YH, Chao WT, Sia KC, Tseng YH and Liu YH: Transient knockdown-mediated deficiency in plectin alters hepatocellular motility in association with activated FAK and Rac1-GTPase. Cancer Cell Int 15: 29-35, 2015.

17 Cheng CC, Chao WT, Liao CC, Tseng YH, Lai YC, Lai YS, Hsu $\mathrm{YH}$ and Liu YH: Plectin deficiency in liver cancer cells promotes cell migration and sensitivity to sorafenib treatment. Cell Adh Migr, 2017. doi: 10.1080/19336918.2017.1288789. [Epub ahead of print]

18 Liu YH, Pei RJ, Cheng CC, Ho HC, Ho CC, Yeh KT, Lee KY and Lai YS: HCC CKs are altered histones during tumor transformation in hepatoma. Res Commun Mol Pathol Pharmacol 112: 27-38, 2002.

19 Ho CC, Cheng CC, Liu YH, Pei RJ, Hsu YH, Yeh KT, Ho LC, Tsai MC and Lai YS: Possible relation between histone 3 and cytokeratin 18 in human hepatocellular carcinoma. In Vivo 22: 457-462, 2008.

20 Barboro P, Alberti I, Sanna P, Parodi S, Balbi C, Allera C and Patrone E: Changes in the cytoskeletal and nuclear matrix proteins in rat hepatocyte neoplastic nodules in their relation to the process of transformation. Exp Cell Res 225: 315-327, 1996.

21 Cheng CC, Lai YC, Lai YS, Chao WT, Tseng YH, Hsu YH, Chen YY and Liu YH: Cell Pleomorphism and Cytoskeleton Disorganization in Human Liver Cancer. In Vivo 30: 549-555, 2016.

22 Buurman R, Gurlevik E, Schaffer V, Eilers M, Sandbothe M, Kreipe H, Wilkens L, Schlegelberger B, Kuhnel F and Skawran B: Histone deacetylases activate hepatocyte growth factor signaling by repressing microRNA-449 in hepatocellular carcinoma cells. Gastroenterology 143: 811-820, 2012.

23 Cheng CC, Liu YH, Lai YC, Hsu YH, Chao WT and Lai YS: Hypoacetylation in association with histone 3 modulation in human hepatocellular carcinoma. In Vivo 29: 237-242, 2015.

24 Farooq M, Hozzein WN, Elsayed EA, Taha NA and Wadaan MA: Identification of histone deacetylase 1 protein complexes in liver cancer cells. Asian Pac J Cancer Prev 14: 915-921, 2013.

25 Sun TY, Xie HJ, Li Z, Kong LF, Gou XN, Li DJ, Shi YJ and Ding YZ: miR-34a regulates HDAC1 expression to affect the proliferation and apoptosis of hepatocellular carcinoma. Am J Transl Res 9: 103-114, 2017.

26 Rikimaru T, Taketomi A, Yamashita Y, Shirabe K, Hamatsu T, Shimada M and Maehara Y: Clinical significance of histone deacetylase 1 expression in patients with hepatocellular carcinoma. Oncology 72: 69-74, 2007.
27 Zhao L, He D, Jiao M, Kong L, Shao C, Chen J, Fang Z, Ma X, Chen H, Li L, Luo S, Zheng N, Chen Y, Wang Q and Fang S: Overexpression of histone deacetylase and amyloid precursor protein in hepatocellular carcinoma. Technol Cancer Res Treat, 2016. pii: 1533034616661664. [Epub ahead of print]

28 Song X, Wang J, Zheng T, Song R, Liang Y, Bhatta N, Yin D, Pan S, Liu J, Jiang H and Liu L: LBH589 Inhibits proliferation and metastasis of hepatocellular carcinoma via inhibition of gankyrin/STAT3/Akt pathway. Mol Cancer 12: 114-126, 2013.

29 Gahr S, Mayr C, Kiesslich T, Illig R, Neureiter D, Alinger B, Ganslmayer M, Wissniowski T, Fazio PD, Montalbano R, Ficker $\mathrm{JH}$, Ocker $\mathrm{M}$ and Quint $\mathrm{K}$ : The pan-deacetylase inhibitor panobinostat affects angiogenesis in hepatocellular carcinoma models via modulation of CTGF expression. Int J Oncol 47: 963-970, 2015.

30 Sun G, Mackey LV, Coy DH, Yu CY and Sun L: The Histone Deacetylase Inhibitor Vaproic Acid Induces Cell Growth Arrest in Hepatocellular Carcinoma Cells via Suppressing Notch Signaling. J Cancer 6: 996-1004, 2015.

31 Liu J, Li G, Wang X, Wang L, Zhao R, Wang J, Kong Y, Ding J, Li J and Zhang L: Droxinostat, a Histone Deacetylase Inhibitor, Induces Apoptosis in Hepatocellular Carcinoma Cell Lines via Activation of the Mitochondrial Pathway and Downregulation of FLIP. Transl Oncol 9: 70-78, 2016.

32 Chen MC, Huang HH, Lai CY, Lin YJ, Liou JP, Lai MJ, Li YH, Teng CM and Yang CR: Novel histone deacetylase inhibitor MPT0G009 induces cell apoptosis and synergistic anticancer activity with tumor necrosis factor-related apoptosis-inducing ligand against human hepatocellular carcinoma. Oncotarget 7: 402-417, 2016

33 Yang H, Lan P, Hou Z, Guan Y, Zhang J, Xu W, Tian Z and Zhang C: Histone deacetylase inhibitor SAHA epigenetically regulates miR-17-92 cluster and MCM7 to upregulate MICA expression in hepatoma. Br J Cancer 112: 112-121, 2015.

34 Hsu FT, Liu YC, Chiang IT, Liu RS, Wang HE, Lin WJ and Hwang JJ: Sorafenib increases efficacy of vorinostat against human hepatocellular carcinoma through transduction inhibition of vorinostat-induced ERK/NF-kappaB signaling. Int J Oncol 45: 177-188, 2014.

35 Park H, Garrido-Laguna I, Naing A, Fu S, Falchook GS, PihaPaul SA, Wheler JJ, Hong DS, Tsimberidou AM, Subbiah V, Zinner RG, Kaseb AO, Patel S, Fanale MA, Velez-Bravo VM, Meric-Bernstam F, Kurzrock R and Janku F: Phase I doseescalation study of the mTOR inhibitor sirolimus and the HDAC inhibitor vorinostat in patients with advanced malignancy. Oncotarget 7: 67521-67531, 2016.
Received April 21, 2017

Revised May 30, 2017

Accepted May 31, 2017 